\title{
Design and analysis of the digital phased antennas characteristics using modern systems of the atomized design (CAD)
}

\author{
Andrey Burykin, Vladimir Kuprits ${ }^{*}$, and Alexander Mescheryakov \\ Tomsk State University of Control Systems and Radioelectronics, Researh Institute of Radio systems, \\ Tomsk, 40 Lenin ave, Russia
}

\begin{abstract}
There are discussed methods of the digital phased antennas design using the modern (computer aided design) CAD. There are given examples of digital antenna blocks and elements design using different CADs.
\end{abstract}

\section{Introduction}

Antenna system is really the first link of the signal space-time processing and therefore it defines characteristics of the system. Nowadays antenna is an electronic system needed a system approach to design. It made to work out the CAD systems meant to design both at the system level and at the level of the antenna separate blocks and elements. So design of the modern antennas suggests to use CAD to improve the efficiency of design and to economize time.

\section{Methods of the digital phased antennas design using CAD}

Digital phased antennas (DPA) are the most effective and perspective systems providing the fast space observation by forming the needed number of rays, many-functional regime and so on. CADs are used to design radar systems, systems of communication and navigation.

The system approach suggests, first the process of DPA organization and development, second, methods of their design and investigation. While using the system approach one should formulate the goal of the DPA design, value it from the problem under consideration point of view. Integration and concretization of the system approach are utilized in such approaches as structural, block-ierarch and object oriented [1]. For example, in case of the structural approach as a kind of the system approach variety it is necessary to synthesize the system variants using components (blocks) and compare variants taking into consideration preliminary prognosed component characteristics [1]. The block-ierarch approach utilize ideas of the objects complex descriptions.

\footnotetext{
*Corresponding author: vladimir.i.kuprits@tusur.ru
} 
This circuit is used to design the system math model and to formulate the demands to the blocks. Then, it is necessary to choose the CAD to perform calculations according to the demands to the DPA.

As an example fig.1 shows the functional circuit of an active DPA with the frequency transformation [2]. This circuit is used to work out the math model and to formulate demands to the blocks, Then it is necessary to choose the CAD allowing to perform calculations answering the demands to the DAP.

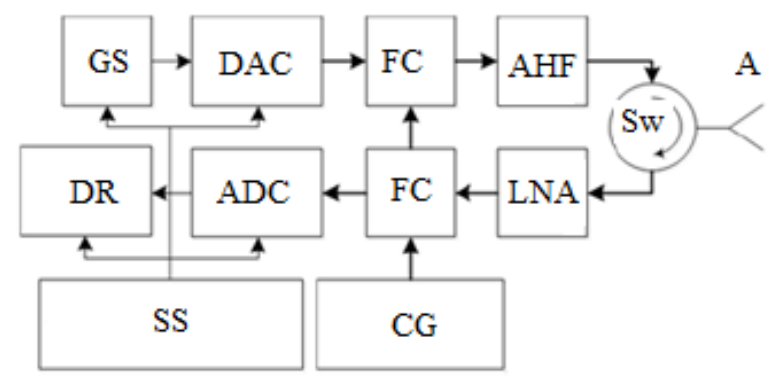

Fig. 1. The functional circuit an active DPA with the frequency transformation. Sw - microwave oven switch; LNA - low noise amplifier; FC - frequency converter; ADC - analog-digital converter; DAC - digital-analog converter; DR - digital receiver; GS - generator of signals; AHF - amplifier of high frequency; A - antenna.

CAD systems CST [3], HFSS [4] and others can be used to calculate the main direction finding characteristics and S-parameters of an antenna elements and the whole antennas.

CST STUDIO SUITE is a group of instruments to design, modeling and optimization three-dimensional, electro-magnetic systems. One of the CST STUDIO SUITE peculiarities is ability to integrate several methods of modeling and modules.

CST STUDIO SUITE includes

CST Microwave studio- instrument to design UHF devices (antennas, filters, feeders and do on);

CST EM Studio - this module is meant to analyze electro-magnetic fields, vortex and surface currents;

CST design studio - instrument for complicated devices decomposition;

Fig. 2 shows a model of a micro strip antenna designed in CST STUDIO SUITE.

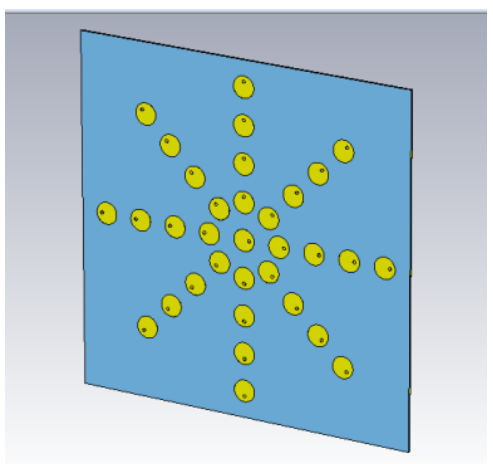

Fig. 2. A model of a micro strip antenna designed in CST STUDIO SUITE.

Nowadays there are different CADs to calculate amplifier, convertor and other nonlinear blocks parameters. So shows fig. 3 [5]. The model was designed at the TUSUR Research Institute of Radio systems and used in some projects. 


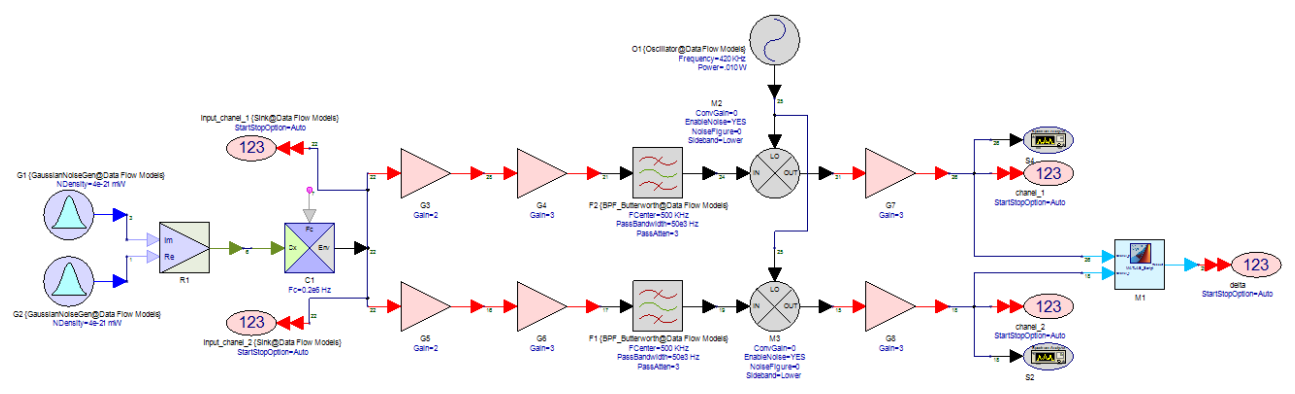

Fig. 3. A model of two receiver-tracts designed in SystemVue.

Fig. 4 shows the calculated signal spectrum at the output of the shown above receiving canal.

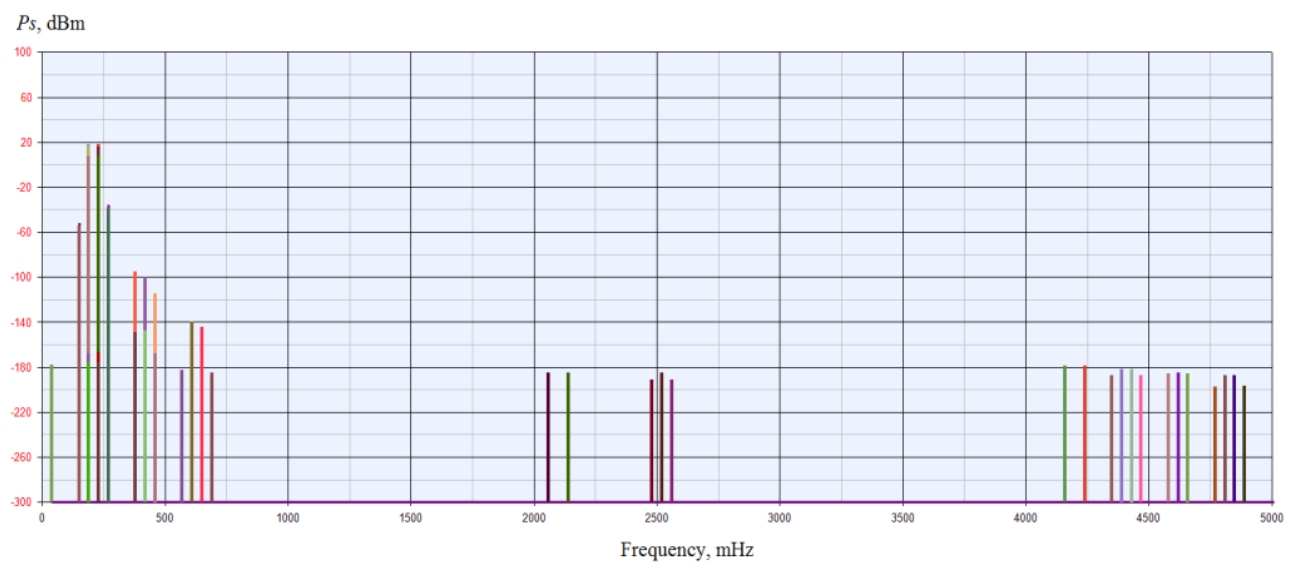

Fig. 4. The calculated signal spectrum at the output of the shown above model receiving canal (CAD SystemVue).

To analyze DPA charateristics it is better to choose Matlab и SystemVue. Matlab (abbreviation of «Matrix Laboratory») is a pocket of programs to solve technical problems [6]. Matlab is a high level language including matrix data structures, many functions, integrated field for design, object-oriented opportunities and program interfaces and algorithms Matlab provides comfortable programming process. Matlab includes specialized pockets of auxiliary programs for design (Simulink, PhasedArraySystemToolbox and so on.) $[6]$.

This CAD is good for the design at the system level. It allows to build a correct model of a DPA. It also allows to make modeling and simulation of all radio systems, subsystems and basic signals.

The advantages of SystemVue are [5]:

- better integration with tests, making faster and easier the radio systems real design based on modeling;

- more convenient interface of modeling, more specialized libraries with opportunity to include own elements;

- opportunity to integrate MATLAB and others.

Fig. 5 shows an example of an antenna model designed in SystemVue.

The system oriented level of the DAP design suggests:

- decomposition into separate blocks in CAD and building a functional circuit,

- design the separate blocks in CAD,

- modeling of the system characteristics using CAD and calculated blocks parameters. 


\section{Template: RF Phased Array (TX)}

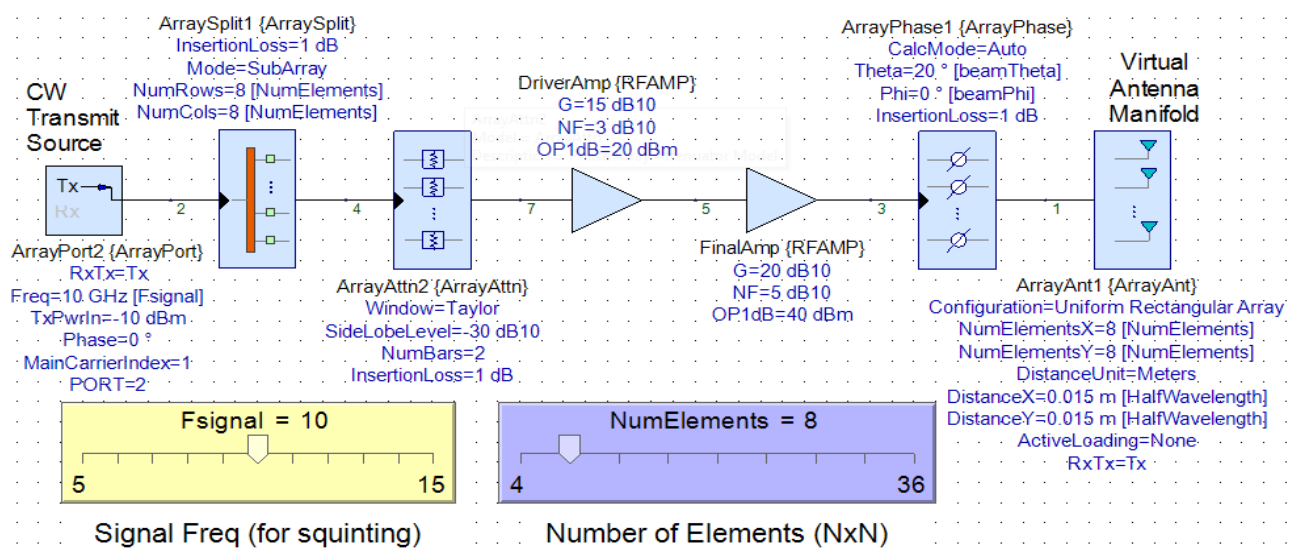

Fig. 5. An example of an antenna model designed in SystemVue.

\section{Conclusion}

An DPA design is a difficult problem needed application of the system approach. It could be performed using modern CADs that allow to solve the problem.

The choice of the proper CAD depends on its ability to integrate calculation results into the system model.

In order to design and integrate DPA at the system level it is advisable to use Matlab and SystemVue. They allow to realize the adequate math model of the system.

The work was performed as part of the state task of the Ministry of Education and Science of the Russian Federation number 8.7348.2017/8.9.

\section{References}

1. Yu. P. Borisov, V. V. Cvetnov, Mathematical modeling of radio systems and devices. Radio i Svyaz, Moscow (1985)

2. D. I. Voskresenskiy, E. V. Ovchinnikova, P. A. Shmachilin, On-board digital antenna arrays and their elements. Radiotehnika, Moscow (2013)

3. Electronic resource: https://www. cst.com/

4. Electronic resource: https://www.ansys.com/

5. Electronic resource: https://www. keysight.com/

6. B. Mahafza, Radar system analysis and design using MatLab. CRC Press (2013) 